\title{
Speaking-club on-line para fortalecer la expresión oral del idioma inglês
}

\section{On-line speaking-club to strengthen English language speaking skills}

DOI: $10.46932 /$ sfjdv2n5-143

Received in: Oct 1st, 2021

Accepted in: Dec 30th, 2021

\section{Maria Isabel Alcazar Cedeño}

Aspirante a Dra. en Educación de la Universidad Cesar Vallejo, Piura-Perú.

Escuela de Posgrado Programa Académico de Doctorado en Educación, Universidad Cesar Vallejo.

Piura-Perú.

Magister en Educación a Distancia y Abierta.

Especialista En Diseño Curricular y Material Educativo Para la Educación A Distancia.

Diploma Superior en Investigación de la Educación a Distancia. Licenciada en Ciencias de la Educación mención Idiomas (Inglés - Francés).

Docente de La Unidad Educativa Nicolás Infante Díaz.

Vicerrectora De La Unidad Educativa a Distancia Ciudad de Quevedo.

Coordinadora Cede-Quevedo Fundación Educación Para el Desarrollo Convenio Con Instituto

Tecnológico Superior Republica de Alemania

Dirección: Quevedo. Vía-Valencia Km 5

Correo Electrónico: maria.alcazar@educacion.gob.ec

\section{Melba Lilian Triana Palma}

Magister en Docencia y Currículo.

Licenciada en Ciencias de la Educación En La Especialización De Inglés Francés.

Certificación en Enseñanza del Idioma Inglés Como Lengua Extranjera - Telf Program.

Docente de la Universidad Técnica de Babahoyo Ext. Quevedo.

Dirección: Babahoyo Ciudadela El Pireo2

Correo Electrónico: Mtriana@Utb.Edu.Ec.

\section{Maria Mercedes Carpio Herrera}

Magister en Docencia y Currículo.

Licenciada En Ciencias de la Educación Mención Idiomas (Inglés - Francés).

Certifícate In Advance English Cefr Level C1 Cambridge. Docente- Coordinadora Técnico Pedagógica de La Unidad Educativa Replica Nicolas Infante Diaz.

Dirección: Quevedo. Av. Walter Andrade ·507 Y San Rafael

Correo Electrónico: Mariacarpio031@gmail.com

\section{Veronica Alexandra Garcia Liscano}

Magister en Docencia y Currículo.

Magister En Pedagogía de los Idiomas Nacionales y Extranjeros Mención en Enseñanza de Inglés.

Licenciada En Ciencias de la Educación Mención Idiomas (Inglés - Francés).

Ingeniera en Marketing.

Docente de Ingles de La Unidad Educativa Rashid Torbay, Playas, Guayas, Ecuador,

Correo Electrónico: Veronicaa.Garcia@Educacion.Gob.Ec 


\title{
RESUMEN
}

En la expresión oral en inglés en estudiantes del nivel secundario, la comunicación oral (speak) es uno de los aspectos más relevantes en el aprendizaje del idioma y es aquí donde se evidencia dificultad al desarrollar una conversación en ingles lo cual implica el desarrollo de destrezas y habilidades para la comunicación. Tiene como objetivo determinar el efecto de la estrategia Speaking Club on-line en el fortalecimiento de la expresión oral en inglés en estudiantes de secundaria de la Institución Educativa Nicolás Infante Díaz. En la construcción de esta estrategia multimedia se consideró antecedentes nacionales e internacionales pertinentes al tema, se fundamenta en la teoría de Chomsky sobre el enfoque comunicativo sintetizado por Oxford (1990).

La investigación es cuantitativa, de diseño cuasi-experimental, El estudio se realizó a una muestra de 70 estudiantes de segundo año de bachillerato el instrumento que se utilizó fue una rúbrica la técnica de análisis de datos fue la prueba no paramétrica la U de Mann Whitney que contrastó la Este trabajo de investigación surgió como respuesta a las dificultades presentadas hipótesis de investigación. Una vez desarrollada la estrategia Speaking Club on-line se evidenció que los estudiantes en el grupo experimental, el $1 \%$ necesita mejorar, el $51 \%$ se encuentra en logro obtenido y $37 \%$ logro notable y alcanza un logro sobresaliente un 9\%. Apreciándose que el grupo experimental tienen un mejor desempeño en la prueba del Post-Test.

Los resultados fueron favorables se logró fortalecer la expresión oral hay una diferencia significativa en el nivel de los estudiantes antes y después de aplicación de la estrategia Speaking Club on-line, con respecto a la contratación de hipótesis general el resultado del sig lateral es $0,003<0.05$ si tiene efectos significativos en el desarrollo de la expresión oral. Por tal razón se recomienda la aplicación de esta estrategia multimedia Speaking Club on-line para ser empleado en otros contextos.

Palabras claves: Fluidez, vocabulario, expresión oral, gramática.

\begin{abstract}
This research work arose as a response to the difficulties presented in oral expression in English in high school students, oral communication (speak) is one of the most relevant aspects in language learning and it is here where difficulty is evident when developing a conversation in English which implies the development of skills and abilities for communication. Its objective is to determine the effect of the online Speaking Club strategy in strengthening oral expression in English in secondary school students from the Nicolás Infante Díaz Educational Institution. In the construction of this multimedia strategy, national and international antecedents relevant to the subject were considered, it is based on Chomsky's theory on the communicative approach synthesized by Oxford (1990).

The research is quantitative, with a quasi-experimental design, The study was carried out on a sample of 70 second-year high school students, the instrument used was a rubric, the data analysis technique was the non-parametric Mann Whitney $U$ test. which contrasted the research hypothesis. Once the online Speaking Club strategy was developed, it was evidenced that the students in the experimental group, $1 \%$ need to improve, $51 \%$ are in achieved achievement and 37\% are remarkable achievement and 9\% reach an outstanding achievement. Appreciating that the experimental group have a better performance in the Post-Test. The results were favorable, it was possible to strengthen oral expression, there is a significant difference in the level of the students before and after the application of the online Speaking Club strategy, with respect to the hiring of general hypotheses, the result of the lateral sig is $0.003<0.05$ if it has significant effects on the development of oral expression. For this reason, the application of this online Speaking Club multimedia strategy is recommended to be used in other contexts.
\end{abstract}

Keywords: Fluency, vocabulary, oral expression, grammar. 


\section{INTRODUCCIÓN}

En la actualidad a nivel mundial comunicarse en inglés es imperioso en diferentes ámbitos como; profesional educativo y laboral, en este contexto, el conocimiento del idioma inglés, la habilidad de poder comunicarse e interactuar es importante para el desarrollo integro de la persona, si damos una mirada a la realidad del idioma inglés en América latina nos daremos cuenta que aún existe la deficiencia en el desarrollo de las competencias comunicacionales en estudiantes pues el dominio del mismo es bajo, pese al aporte que fomenta las tecnologías de información y comunicación en el desarrollo del lenguaje ya sea oral o escrito, pero no se refleja avance en las competencias comunicativas.

A nivel Nacional en Ecuador el Ministerio de Educación, en el año (2016) uno de los principales objetivos del currículo se basa en desarrollar la comunicación de los estudiantes que pueda interactuar y comunicarse pues la realidad que viven los estudiantes es distinta, no pueden comunicarse con otros hablantes del idioma inglés, muestran deficiencia en desarrollar diálogos básicos por lo tanto es inminente que debe existir cambios en las estrategias utilizadas por los docentes para enseñar en Idioma Inglés que promueva al desarrollo de la producción oral.

Cabe agregar que Ecuador y sus leyes educativas ha promovido estándares hacía la educación de calidad, entre las que se destaca el diseño de currículo de lengua inglesa, sin embargo, Ecuador ocupa el lugar más bajo de los países evaluados, de 100 se registra en el 81 avo lugar, según informe estandarizado del Marco Común Europeo significa entonces es emergente realizar cambios que mejoren los dominios del idioma inglés.

A nivel local en el distrito 12D03 Quevedo-Mocache, los resultados de los estudiantes presentan problemas en cuanto a los niveles de competencia lingüística, Siendo la producción oral la que presenta mayor grado de complejidad que tienen estudiantes al desarrollar como por ejemplo son incapaces de comunicarse correctamente.

Debido a esto es importante analizar la metodología y estrategias utilizadas por los docentes en efecto aplica estrategias digitales innovadoras (speaking club). Es por esta razón que se realizó este estudio para mejorar la expresión oral del inglés.

Es innegable que nos encontramos frente a tiempos donde los estudiantes y docentes están altamente involucrados con la tecnología en el proceso de aprendizaje en base a esa realidad los docentes deben corresponder con estrategias pedagógicas interactivas innovadas que despierte el interés motive al estudiante y sobre todo logren alcanzar su nivel del Idioma Inglés para poder comunicarse efectivamente y el rendimiento académico no sea un inconveniente en el estudiante. Ante lo expuesto surge la inquietud de investigar sobre: ¿Cuál es el efecto de Speaking-Club on-line como estrategia multimedia en la expresión oral del idioma Inglés en los estudiantes de educación básica y bachillerato del Distrito 12D03 


\section{Quevedo-Mocache?}

Desde el punto de vista teórico se basó en los aportes de J. Willis (2015). Thornbury (2015) dado que el programa Speaking Club On-Line radica en elevar los conocimientos del inglés con énfasis a la producción oral insertarlos en entornos virtuales mediante el uso de plataformas digitales que corresponden a espacios de conversación como estrategia multimedia para fortalecer la producción oral del Inglés. Por otro lado, hace referencia a las reflexiones sobre la producción oral planteada por Chomsky, (1965). Además, el programa Club de conversación delimita las habilidades del idioma escucha y habla como leer y escribir, planteadas por Oxford (1990).

En este sentido se plantea como objetivo general: Demostrar el efecto de la implementación del Speaking-Club on-line como estrategia multimedia en la expresión oral del idioma Inglés en los estudiantes de educación básica y bachillerato del Distrito 12D03 Quevedo-Mocache. Asimismo, se plantea como objetivos específicos: Identificar los niveles de aprendizaje de los estudiantes en la expresión oral del idioma Ingles del Distrito 12D03 Quevedo-Mocache, antes de la implementación del Speaking-Club online como estrategia multimedia; de la misma manera, Evaluar los niveles de aprendizaje de los estudiantes, en la expresión oral del idioma Inglés del Distrito 12D03 Quevedo- Mocache, después de la implementación del Speaking-Club on-line como estrategia multimedia.

También se plantea establecer las diferencias del nivel de expresión oral del idioma inglés entre los grupos experimental y control después de la implementación del Speaking-Club on-line como estrategia multimedia y, por último, Establecer las diferencias del nivel de expresión oral del idioma Inglés en el grupo experimental después de la implementación del Speaking-Club on-line como estrategia multimedia.

\section{MARCO TEORICO}

Anampa (2019) en la Universidad ULADECH en este estudio como objetivo general se planteó determinar el efecto de lectura intensiva en la comprensión lectora en inglés estudiantes, el investigador plantea buscar estrategias para mejorar el aprendizaje del idioma inglés...Este estudio se puede ver que en la actualidad se requiere que los docentes encargados de orientar y enseñar el idioma Inglés, implemente estrategias que respondan a mejorar las deficiencias de los niveles de inglés sobre todo las habilidad de comunicarse (speaking) que son fundamentales.

De igual manera Muñoz (2019) en su investigación doctoral, Programa TPACK para mejorar competencias lingüísticas del idioma Inglés, utilizando enfoque cuantitativo, de diseño cuasi experimental, se hizo este estudio con muestra de 48 estudiantes de los resultados obtenidos,...como se puede entender en el proceso de aprendizaje del Idioma Inglés es importante que los estudiantes desarrollen la competencia oral, saber la estructura lingüística y como emplearla es conveniente utilizar estrategias multimedia con 
enfoque comunicativo y lograr una mejor comprensión en los estudiantes.

También referimos en sus investigaciones a Caicedo (2017) en su trabajo de investigación en Ecuador en la Universidad Central, Speaking Card mejora significativamente la producción oral,...los resultados de este estudio evidencio que los estudiantes mejoran su dominio de las destrezas en el aprendizaje del idioma inglés usando speaking card como estrategia de aprendizaje.

Esto demuestra que la aplicación de herramientas interactivas si mejoran el nivel de producción oral siendo esto fundamental para poder tener una conversación.

Es importante mencionar, que, para mejorar la comunicación oral del idioma inglés, es necesario se trabaje de forma colaborativa teniendo como herramientas imprescindiblesherramientas interactivas que faciliten el aprendizaje, la participación será más productiva, tendrán más confianza; para ello debemos tener claro los enfoque, las teorías,tales como estrategias.

En cuanto al método que se fundamenta la enseñanza del idioma Inglés, es el método audiolingual, el mismo que da valor a la producción oral y la parte de la escucha, su objetivo es tener el dominio del idioma inglés mediante ejercicios de imitación de los patrones nativos.

En este contexto es importante mencionar el Speaking club-online representa un aspecto fundamental en el aprendizaje del idioma inglés, porque es un medio que permite interactuar con otras personas, genera confianza en el estudiante, la practicapermite la adquisición de fluidez en el idioma Inglés.

Las dimensiones propuestas para la variable Speaking Club on-line es aquel que a través del cual se propone adquirir la destreza productiva en la enseñanza como lengua extranjera implica fluidez. La dimensión Práctica de conversación comprende: personas capaces de almacenar, procesar y transmitir información.Siendo Speaking Club on-line una herramienta que tiene una amplia mirada y enfatiza los tipos de conocimientos: Pedagógico, tecnológico la tecnología tiene gran influencia en todos los ámbitos pues la combinación entre la tecnología y pedagogía en el proceso d enseñar en idioma Inglés es indispensable que se dé una relación activa, entre los componentes del conocimiento en contextos de aprendizaje.

En la presente investigación, se va reflejar este tipo de conocimiento en el docente cuando se observen en el pre test y post test en los estudiantes que pasen de un nivel a do demuestren las habilidades de comunicarse efectivamente en el idioma Inglés, muestren interés por aprender el y también se sientan motivados y sobre todo respondieron eficientemente los test.

Mientras que el conocimiento pedagógico en Speaking Club on-line es el saber que tienelos docentes en relación con los métodos y técnicas aplicar en el proceso de enseñanzadel idioma inglés. Considerando como base los fines de la educación en un marco de principios y valores educativos para 
una educación Integral, pues con la Implementación de Speaking Club on-line como herramienta interactiva para mejorar la producción oral en la enseñanza Ingles aspira que los estudiantes adquieran habilidades y destreza paracomunicarse con fluidez.

\section{METODOLOGÍA}

La investigación presenta un enfoque cuantitativo, el método hipotético-deductivo considerado por los investigadores como una herramienta para describir una realidad desde su percepción, donde debe generar hipótesis en un intento de solucionar problemas o cambiar la realidad.

Este estudio es aplicado tiene la perspectiva de abordar problemas que demandan solución o cambiar la realidad, en este sentido la investigación tiene una aplicación inmediata que permite realizar mediciones y determinar la efectividad del Speaking Club on-line en mejorar el nivel del idioma inglés, por su desarrollo es de tipo cuantitativa.

Es una estrategia multimedia que tiene como base la utilización de herramientas tecnológicas ideal para alcanzar el nivel de fluidez en el idioma, esta herramienta permite que las destrezas de idioma sean desarrolladas de forma interactiva y dinámica además ayuda a la motivación, Martin (1992) menciono que club de conversación (speak) es una manera de llevar diferente contexto al aula.

La expresión oral se define como un conjunto de técnicas que permiten que las personas interactúen por medio del lenguaje oral, para desarrollar la interacción se requiere conocer las herramientas del lenguaje en consecuencia el aprendizaje de una lengua extranjera debe tener en cuenta este aspecto, quien desee adquirir un nuevo idioma debe desarrollar competencia de emitir y receptar mensaje. Luna y Sanz, 2003)

\subsection{POBLACIÓN, MUESTRA, MUESTREO Y UNIDAD DE ANÁLISIS}

En este estudio de acuerdo con los datos proporcionados la población estuvo constituida por 140 estudiantes del nivel de bachillerato de ambos sexos con edades entre de 15 a 17 por Instituciones Educativas del Distrito 12D03 Quevedo, la misma que está organizada de la siguiente manera:

Tabla 1. La población de la Investigación:

\begin{tabular}{ll}
\hline Secciones & Estudiantes \\
\hline 2nd & 35 \\
2nd & 35 \\
2nd & 35 \\
& 35 \\
\hline & 140 \\
\hline
\end{tabular}


Tabla 2. Muestra de estudio

\begin{tabular}{lll}
\hline Grupos & Paralelos & Total \\
\hline G-Experimental & Second A & 35 \\
G-Control & Second B & 35 \\
\hline & & 70 \\
\hline
\end{tabular}

\subsection{CRITERIOS DE SELECCIÓN}

Criterios de inclusión: Estudiantes de la Unidad Educativa Nicolás Infante Díaz que se inscribieron en el programa de Speaking Club on-line

- Estudiantes que tengan registro de matrícula reglamentaria en el periodo 2021

- Carta de autorización de los representantes legales de estudiantes.

- Modelo de carta de aceptación parte de los estudiantes.

\subsection{CRITERIOS DE EXCLUSIÓN}

- Estudiantes de la Unidad Educativa Replica Nicolás con estudios previos en cursos estudiantes con certificaciones internacionales.

- Los participantes que no cuenten con la autorización de los PP.FF.

- Participantes que no cuenten con recursos a internet.

El muestreo no probabilístico, cuando todos los miembros de la población no tienen la misma oportunidad de ser incluidos.

En este caso la unidad de análisis está constituida por los estudiantes que están en el nivel de bachillerato

Tabla 3. Rubrica de medición nivel idioma inglés énfasis a la expresión oral Need improvement sufficient archieved Notably archieved Oustandingly archieved

\begin{tabular}{llllll}
\multicolumn{2}{l}{ Necesita mejora } & logro alcanzado & logro notable & \multicolumn{2}{l}{ logro sobresaliente } \\
\hline Fluidez & (Frases) & $0-2$ & $3-4$ & $5-6$ & $7-8$ \\
\hline Pronunciación & (items) & $0-2$ & $3-4$ & $5-6$ & $7-8$ \\
\hline Gramática (estructura oraciones) & $0-2$ & $3-4$ & $5-6$ & $7-8$ \\
\hline Vocabulario & (objetos) & $0-2$ & $3-4$ & $5-6$ & $7-8$
\end{tabular}

La confiabilidad de la rúbrica expresión oral será lograda por medio de las derivaciones que brinda sus items con opciones de contestación ( $1=$ correcto y $0=$ incorrecto ) para la validez del instrumento se someterá a juicio de expertos puesto que determinan su aplicabilidad e idoneidad y se seguirá las recomendaciones, para determinar la confiabilidad se utilizara la formula KR 20 
Tabla 4. Evidencia de validez de contenido a través de juicio de expertos

\begin{tabular}{llll}
\hline EXPERTO & GRAD & RESULTA \\
& O & DO & \\
\hline Gonzalo Peñafiel Nivela & & Doctor & Aplicable \\
Flor Carriel & Doctor & Aplicable \\
Blanca Aracely Auria. & Doctor & Aplicable \\
\hline
\end{tabular}

\subsubsection{Evidencia de confiabilidad del instrumento}

Un instrumento es confiable cuando se realizan diferentes mediciones con significativas variaciones en este estudio se realizó verificación y confiabilidad del instrumento de la rúbrica con escala de mediciones del nivel de la expresión oral, después de aplicar el instrumento los datos se registraron en un Excel y a través de la prueba KR20 se obtuvo resultado 0,765 lo que evidencia que hay confiabilidad en el instrumento después fue aplicado el pre-test y post-test.

Tabla 5. Evidencia de confiabilidad de la escala de medición

\begin{tabular}{lll}
\hline KR 20 & Elementos \\
\hline
\end{tabular}

Rubrica de medición de nivel

\section{0,765}

\section{RESULTADOS}

OE.1: Identificar los niveles de aprendizaje de los estudiantes en la expresión oral del idioma Inglés del Distrito 12D03 Quevedo-Mocache, antes de la implementación Speaking-Club on-line como estrategia multimedia.

TABLA 6: Nivel de la expresión oral en el grupo experimental y control según en pretest

\begin{tabular}{ccccc} 
EXPRESION ORAL & \multicolumn{2}{c}{ GRUPO CONTROL } & \multicolumn{2}{c}{$\begin{array}{c}\text { GRUPO } \\
\text { EXPERIMENTAL }\end{array}$} \\
\cline { 2 - 5 } & FR & de columna del N & FR & de columna del N \\
\hline LOGRO & 0 & $0,0 \%$ & 0 & $0,0 \%$ \\
SOBRESALIENTE & 1 & $2,9 \%$ & 5 & $14,3 \%$ \\
LOGRO NOTABLE & 4 & $11,4 \%$ & 1 & $2,9 \%$ \\
NECESITA MEJORAR & 30 & $85,7 \%$ & 29 & $82,9 \%$ \\
\hline Total & 35 & $100,0 \%$ & 35 & $100,0 \%$
\end{tabular}

Fuente: Base de dato obtenido en la aplicación del documento

Prom: Promedio / Dif: Diferencia.

Salida: SPSS Vrs. 25.0

De los resultados mostrados en el pre-test, se distribuye Expresión oral con un 82,9\% en el grupo experimental en nivel necesita mejora, en 2,9 logro obtenido y en logro notable el 14,3\%. De la misma manera podemos observar los resultados del grupo de control con un 85,7\%, necesita mejorar, se ubica un $11 \%$ en logro obtenido y el $2,9 \%$ en logro sobresaliente. Este análisis muestra indicios de una 
homogeneidad de la expresión oral del inglés ambos grupos antes de la aplicación de la donde se encuentran los estudiantes en el nivel necesita mejorar y un mínimo porcentaje logro alcanzado y logro notable.

FIGURA 1

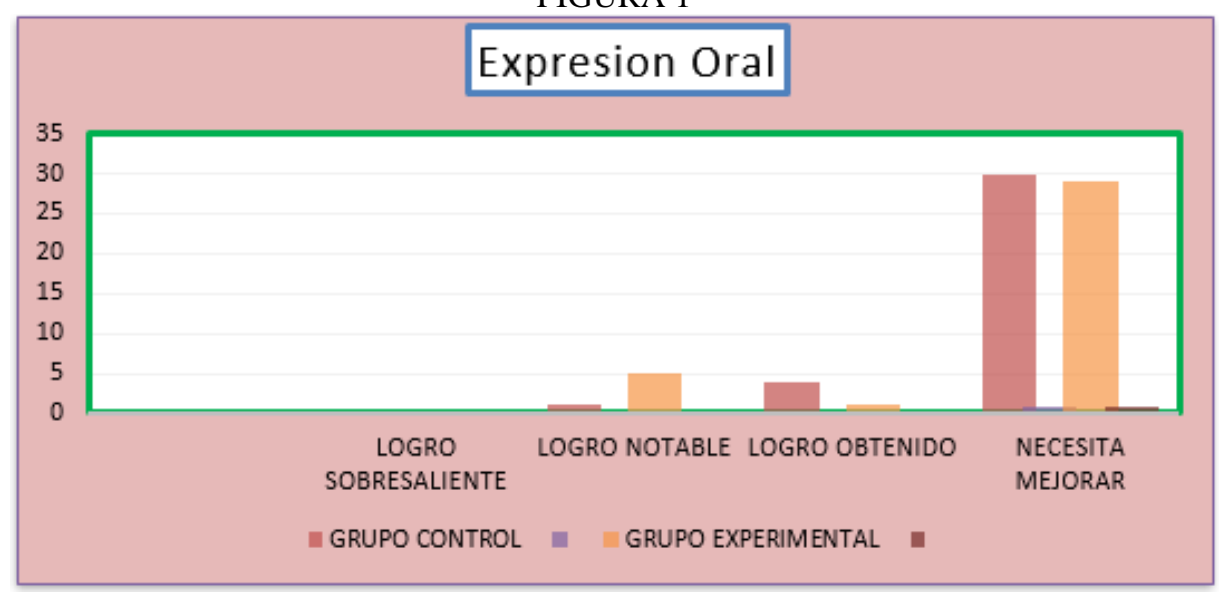

OE.2: Evaluar los niveles de aprendizaje de los estudiantes, en la expresión oral del idioma Ingles del Distrito 12D03 Quevedo- Mocache, después de la implementación del Speaking-Club on-line como estrategia multimedia.

Tabla 7. Niveles expresión oral en inglés prueba post test.

\begin{tabular}{lllll}
\multirow{2}{*}{ Exal } & EXPresión & \multicolumn{3}{l}{ CONTROL } \\
\cline { 2 - 5 } & $\mathrm{n}$ & $\%$ & $\mathrm{n}$ & $\%$ \\
\hline NECESITA & 1 & $3,00 \%$ & 28 & $80,00 \%$ \\
MEJORAR & 18 & $51 \%$ & 4 & $11,00 \%$ \\
LOGRO & 13 & $37,00 \%$ & 2 & $6,00 \%$ \\
$\begin{array}{c}\text { OBTENIDO } \\
\text { LOGRO }\end{array}$ & 3 & $9,00 \%$ & 1 & $3,00 \%$ \\
$\begin{array}{c}\text { NOTABLE } \\
\text { LOGRO }\end{array}$ & 3 & $100,00 \%$ & 35 & $100,00 \%$ \\
SOBRESALIENTE & 35 & & &
\end{tabular}

Figura 2

\section{EXPRESION ORAL}

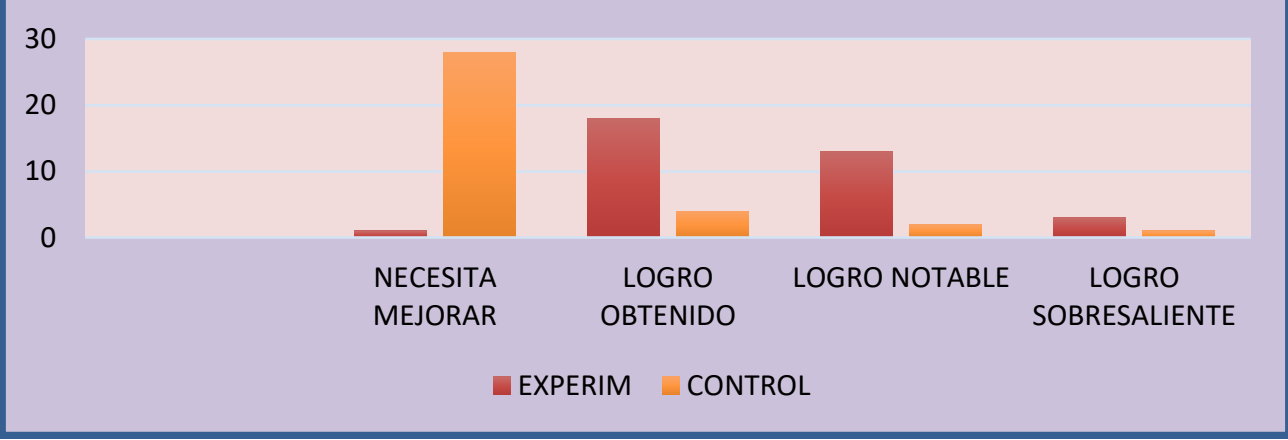


Los resultados de la prueba pos test para ambos grupos, compuesta por 70 estudiantes sobre la variable expresión oral en inglés por cada grupo se visualiza que en el grupo control el $80 \%$ está en inicio, necesita mejorar, un $11 \%$ están proceso , en logro obtenido, están un $9 \%$ y logro notable un $6 \%$ y alcanza logro sobresaliente un 3\% . En cambio, en el grupo experimental, el 1\% está en inicio necesita mejorar, el $51 \%$ en logro obtenido y $37 \%$ logro notable y alcanza un logro sobresaliente un $9 \%$. Se aprecia que después de la aplicación del programa Speaking Club on-line el grupo experimental tiene mejor desempeño así en resultados de la prueba del post-test.

Tabla 8: Niveles de las dimensiones exp.oral del ingles

\begin{tabular}{|c|c|c|c|c|c|c|c|}
\hline & GRO SOBRESALI & NTE & $\begin{array}{l}\text { LOC } \\
\text { NOT }\end{array}$ & & $\begin{array}{c}\text { LOGRO } \\
\text { OBTENIDO } \\
\end{array}$ & $\begin{array}{l}\text { NEC } \\
\text { MES }\end{array}$ & $\begin{array}{l}\text { SITA } \\
\text { RAR }\end{array}$ \\
\hline \multirow{8}{*}{ EXPERIMENTAL } & \multirow{2}{*}{\multicolumn{2}{|c|}{ FLUIDEZ }} & 4 & 3 & 3 & 25 & 35 \\
\hline & & & 11,00 & 9,00 & 9,00 & 71,00 & 100,00 \\
\hline & & - & 4 & 5 & 3 & 23 & 35 \\
\hline & \multicolumn{2}{|l|}{ PRONUNCIACION } & 11,00 & 14,00 & 9,00 & 66,00 & 100,00 \\
\hline & \multirow{2}{*}{\multicolumn{2}{|c|}{ VOCABULARIO }} & 5 & 7 & 1 & 22 & 35 \\
\hline & & & 14,00 & 20,00 & 3,00 & 63,00 & 100,00 \\
\hline & \multirow{2}{*}{\multicolumn{2}{|c|}{ GRAMATICA }} & 5 & 3 & 6 & 21 & 35 \\
\hline & & & 14,00 & 9,00 & 17,00 & 60,00 & 100,00 \\
\hline & \multirow{2}{*}{\multicolumn{2}{|c|}{ FLUIDEZ }} & 4 & 4 & 3 & 24 & 35 \\
\hline & & & 11,00 & 11,00 & 9,00 & 69,00 & 100,00 \\
\hline & \multirow{2}{*}{\multicolumn{2}{|c|}{ PRONUNCIACION }} & 3 & 4 & 6 & 22 & 35 \\
\hline & & & 9,00 & 11,00 & 17,00 & 63,00 & 100,00 \\
\hline & VOCABULARIO & & 4 & 5 & 3 & 23 & 35 \\
\hline \multirow{3}{*}{ CONTROL } & & & 11,00 & 14,00 & 9,00 & 66,00 & 100,00 \\
\hline & \multirow{2}{*}{\multicolumn{2}{|c|}{ GRAMATICA }} & 4 & 4 & 6 & 21 & 35 \\
\hline & & & 12,00 & 11,00 & 17.00 & 60,00 & 100,00 \\
\hline
\end{tabular}

Interpretación: En la tabla 4 y figura 3, el resultado del post-test en la dimensión de la fluidez del idioma ingles los estudiantes del grupo experimental se encuentran el $71 \%$ necesita mejorar, en nivel de la pronunciación se ubican en un $66 \%$ necesita mejorar, en el nivel de vocabulario un $66 \%$ necesita mejorar y por último en nivel de la gramática llegaron a un $60 \%$ necesita mejorar.

Figura 3

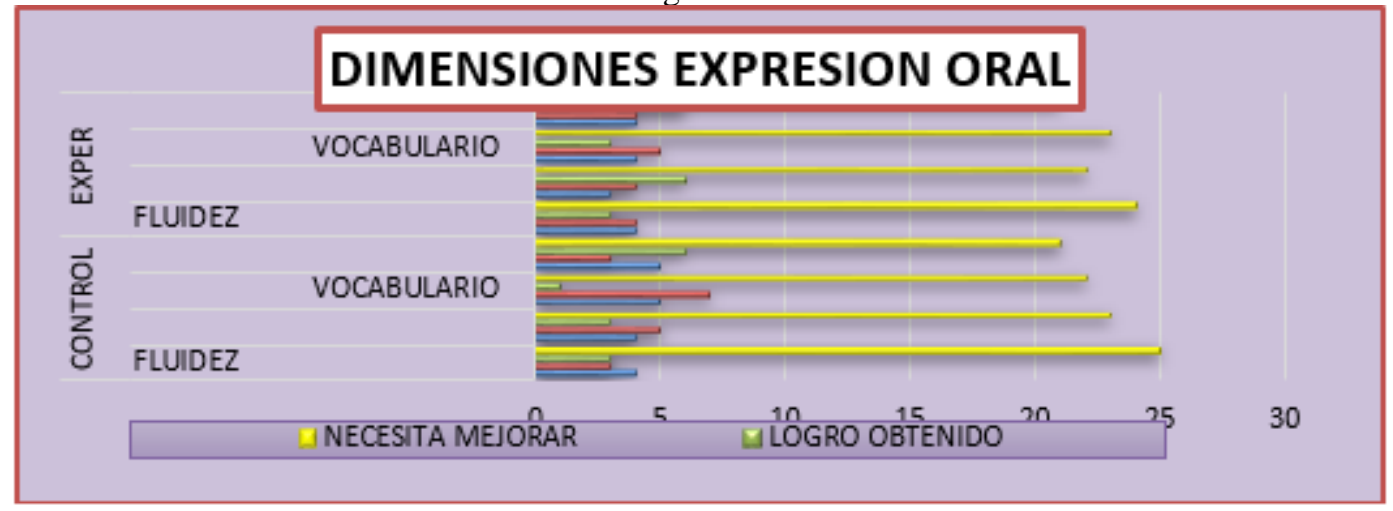


Tabla 9: Niveles de las dimensiones de la expresión oral del idioma inglés del grupo experimental y control después de la aplicación de Speaking Club On-line como estrategia multimedia.

\section{NIVELES DEL IDIOMA INGLES DESPUES DEL POST-TEST}

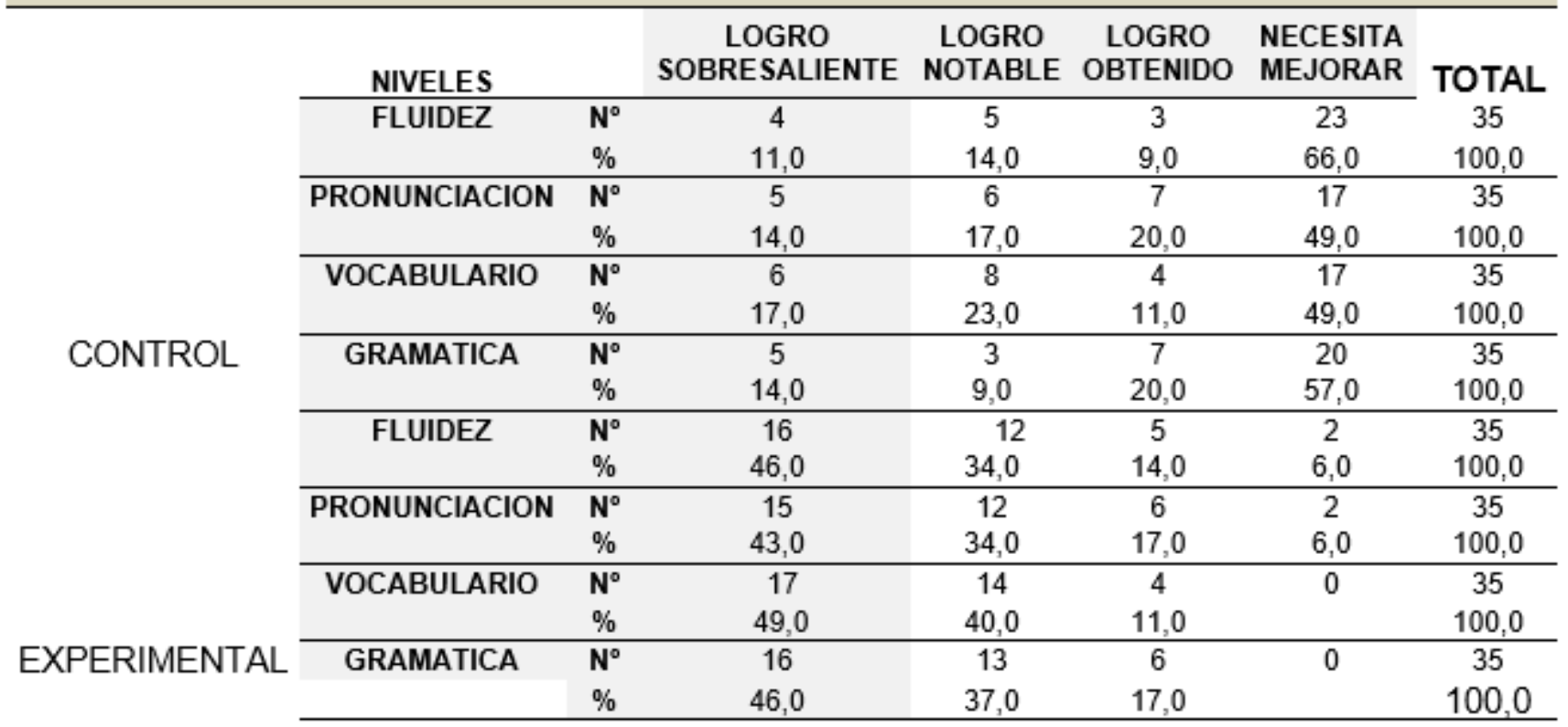

Interpretación: el resultado del post-test en la dimensión de la expresión oral del idioma ingles los estudiantes del grupo control el $66 \%$ se encuentran en nivel que necesita mejorar el $9 \%$ en logro obtenido, el 14\% logro notable y un $11 \%$ en logro sobresaliente, en nivel de la pronunciación se ubican en un $49 \%$ necesita mejorar, el $20 \%$ el logro obtenido, el $17 \%$ logro notable y $11 \%$ en logro obtenido, el $23 \%$ en logro notable y el $17 \%$ en logro sobresaliente, en nivel de la gramática el $57 \%$ necesita mejorar, el 20\% logro obtenido, el 95\% logro notable y el 14\% logro sobresaliente por otro lado tenemos al grupo experimental en la dimensión de la fluidez del idioma ingles los estudiantes el $46 \%$ se encuentran en nivel de logro sobresaliente, el $34 \%$ en nivel de logro notable el 14\% llegaron a nivel de logro obtenido y el $6 \%$ aun necesitan mejorar, en pronunciación se ubican el $43 \%$ logro sobresaliente, el 34\% en logro notable, el 17\% llegaron a logro obtenido y un $6 \%$ aún necesita mejorar, en vocabulario el $49 \%$ llegaron a un nivel de logro sobresaliente, el $40 \%$ logro notable, el 11\% logro obtenido, en la gramatica el $46 \%$ se ubican en logro sobresaliente el $37 \%$ logro notable, el $17 \%$ logro obtenido. Demostrándose que, después de aplicar Speaking Club on.line como estrategia multimedia para la enseñanza del idioma ingles en los estudiantes del grupo experimental presentan una mejora significativa en comparación al grupo control en sus cuatro dimensiones, ya que en el grupo experimental la mayoría de los estudiantes se ubican en Logro Sobresaliente y Logro Notable teniendo ventaja del grupo control donde los estudiantes se ubican en el nivel En inicio necesitan mejorar porque no mejoraron en la expresión Oral del idioma ingles . 
Grafico. 4

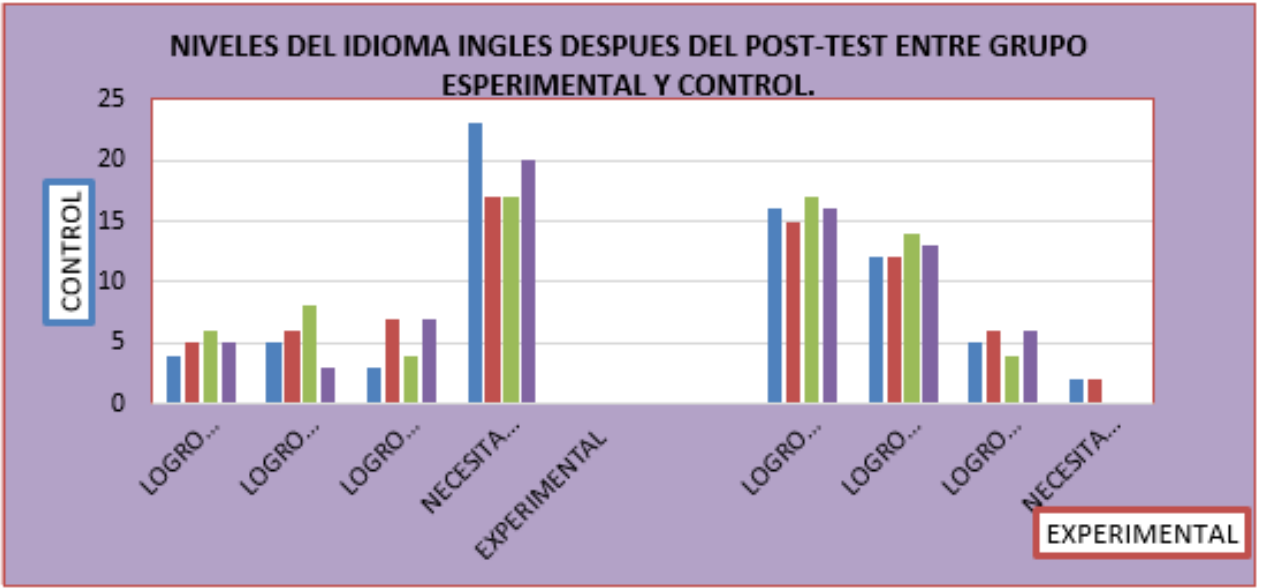

Tabla 10 Nivel de fluidez del idioma ingles en segundo bachillerato de nivel secundaria de la Institución Educativa Nicolás Infante Díaz, Quevedo, 2020

\begin{tabular}{|c|c|c|c|c|c|c|c|c|}
\hline \multirow{2}{*}{ FLUIDEZ } & \multicolumn{2}{|c|}{ G.C PRE-TEST } & \multicolumn{2}{|c|}{$\begin{array}{l}\text { GRUPO EXP. PRE- } \\
\text { TEST }\end{array}$} & \multicolumn{2}{|c|}{ G.EXP. P.TEST } & \multicolumn{2}{|c|}{ G.CONT. POST-TEST } \\
\hline & $\mathrm{n}$ & $\%$ & $\mathrm{n}$ & $\%$ & $\mathrm{n}$ & $\%$ & $\mathrm{n}$ & $\%$ \\
\hline $\begin{array}{l}\text { LOGRO } \\
\text { SOBRESALIENTE }\end{array}$ & 0 & $0,00 \%$ & 0 & $0,00 \%$ & 3 & $9,00 \%$ & 1 & $3,00 \%$ \\
\hline LOGRO NOTABLE & 1 & $2,90 \%$ & 5 & $14,30 \%$ & 13 & $37,00 \%$ & 2 & $6,00 \%$ \\
\hline $\begin{array}{l}\text { LOGRO } \\
\text { OBTENIDO }\end{array}$ & 4 & $11,40 \%$ & 1 & $2,90 \%$ & 18 & $51 \%$ & 4 & $11,00 \%$ \\
\hline $\begin{array}{l}\text { NECESITA } \\
\text { MEJORAR }\end{array}$ & 30 & $85,70 \%$ & 29 & $82,90 \%$ & 1 & $3,00 \%$ & 28 & $80,00 \%$ \\
\hline Total & 35 & $100,00 \%$ & 35 & $100,00 \%$ & & $100,00 \%$ & & $100,00 \%$ \\
\hline
\end{tabular}

\subsection{DESCRIPCIÓN}

Los resultados de la prueba pos test para ambos grupos, compuesta por 70 estudiantes sobre la variable expresión fluidez del idioma inglés por cada grupo se visualiza que en el grupo control el 85,70\% está en inicio, necesita mejorar, un 11,40\% están proceso, en logro obtenido, están un 2\% y logro notable, el grupo experimental antes de la aplicación de la estrategia Speaking Club on-line 82,90\% está en inicio, necesita mejorar, un 2,90\% están proceso , en logro obtenido, están un 14\% y logro notable . En cambio el grupo control después de la aplicación de la estrategia Speaking Club $80 \%$ se encuentra en necesita mejorar el $11 \%$ en logro obtenido el 6\% en logro notable, el grupo experimental 3\% está en inicio necesita mejorar, un $51 \%$ se ubicó en logro notable y $51 \%$ en logro obtenido Se aprecia que después de la aplicación del programa Speaking Club on-line el grupo experimental tiene mejor desempeño así en resultados de la prueba del post-test. 
GRAFICO 5

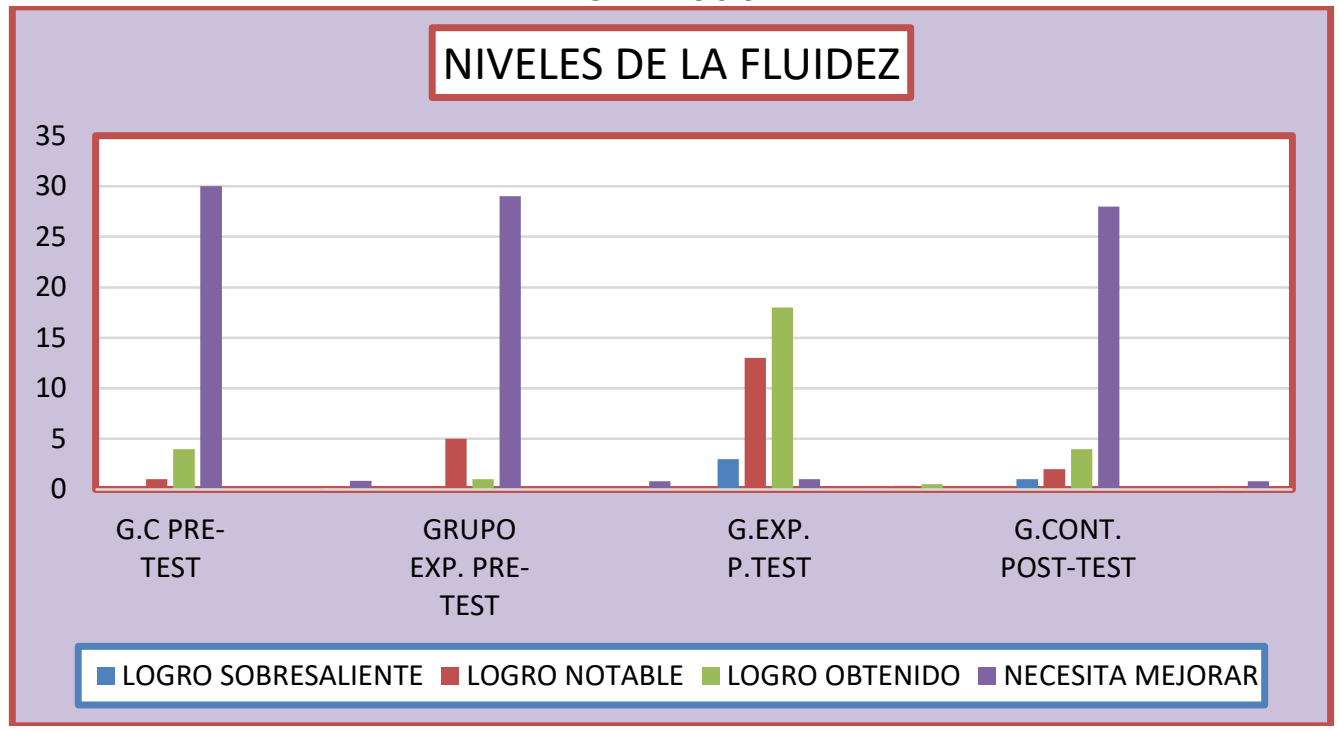

Tabla.11 Nivel de la pronunciación del idioma inglês

Resultados variable pronunciacion en inglés prueba pre test

\begin{tabular}{lcccccccc}
\hline \multirow{2}{*}{ PRONUNCIACION } & \multicolumn{2}{c}{ G.C PRE-TEST } & \multicolumn{2}{c}{$\begin{array}{c}\text { GRUPO EXP. } \\
\text { PRE-TEST }\end{array}$} & \multicolumn{2}{c}{ G.EXP. P.TEST } & \multicolumn{2}{c}{$\begin{array}{c}\text { G.CONT. POST- } \\
\text { TEST }\end{array}$} \\
\cline { 2 - 10 } & $\mathrm{n}$ & $\%$ & $\mathrm{n}$ & $\%$ & $\mathrm{n}$ & $\%$ & $\mathrm{n}$ & $\%$ \\
\hline LOGRO & 0 & - & 0 & $0 \%$ & 4 & $11 \%$ & 0 & $0 \%$ \\
SOBRESALIENTE & 1 & 0,03 & 5 & $14 \%$ & 30 & $86 \%$ & 4 & $11 \%$ \\
LOGRO NOTABLE & 1 & 0,11 & 1 & $3 \%$ & 1 & $3 \%$ & 1 & $3 \%$ \\
LOGRO & 4 & 0,86 & 29 & $83 \%$ & 0 & $0 \%$ & 30 & $86 \%$ \\
OBTENIDO & 30 & 1,00 & 35 & $100 \%$ & 35 & $100 \%$ & 35 & $100 \%$ \\
NECESITA & 35 & & & & & & & \\
MEJORAR & & & & & & & &
\end{tabular}

\subsection{INTERPRETACION}

Los resultados de la pronunciación pos test para ambos grupos, compuesta por 70 estudiantes sobre la variable expresión oral en inglés por cada grupo se visualiza que en el grupo control el 86,\% está en inicio, necesita mejorar, un 11,00 \% están proceso, en logro obtenido, están un 3\%, el grupo experimental antes de la aplicación de la estrategia Speaking Club on-line 83,\% está en inicio, necesita mejorar, un 3\% están proceso, en logro obtenido, están un 14\% y logro notable. En los resultados del grupo control del post-test después de la aplicación de la estrategia multimedia Speaking Club online , el 83\% aun necesita mejorar y el en logro obtenido y $3 \%$ logro notable, en cambio en grupo experimental $3 \%$ está en inicio necesita mejorar, un $86 \%$ se ubicó en logro notable y $11 \%$ en cambio en los resultados del grupo experimental $11 \%$ se encuentran en logro sobresaliente, a partir de estos resultados se aprecia que después de la aplicación de la estrategia Speaking Club on-line el grupo experimental tiene mejor desempeño así en resultados de la prueba del post-test. 
Tabla.12 Nivel de la gramática del idioma ingles

\begin{tabular}{|c|c|c|c|c|c|c|c|c|}
\hline \multirow[t]{2}{*}{ GRAMATICA } & \multicolumn{2}{|c|}{ CONT. P-TEST } & \multicolumn{2}{|c|}{ EXPERIMENTAL } & \multicolumn{2}{|c|}{ CONT. POST.T } & \multicolumn{2}{|c|}{ EXPERIMENTAL } \\
\hline & $\mathrm{n}$ & $\%$ & n & $\%$ & $n$ & $\%$ & $\mathrm{n}$ & $\%$ \\
\hline $\begin{array}{l}\text { ECESITA } \\
\text { IEJORAR }\end{array}$ & 27 & $71 \%$ & 28 & $80 \%$ & 21 & $60 \%$ & 1 & $3 \%$ \\
\hline $\begin{array}{l}\text { LOGRO } \\
\text { OBTENIDO }\end{array}$ & 7 & $20 \%$ & 3 & $8 \%$ & 10 & $28 \%$ & 19 & $54 \%$ \\
\hline LOGRO NOTABLE & 1 & $2,90 \%$ & 4 & $11,40 \%$ & 2 & $6,00 \%$ & 12 & $34,00 \%$ \\
\hline $\begin{array}{l}\text { LOGRO } \\
\text { SOBRESALIENTE }\end{array}$ & 0 & $0,00 \%$ & 0 & $0,00 \%$ & 2 & $6,00 \%$ & 3 & $9,00 \%$ \\
\hline
\end{tabular}

GRAFICO 7

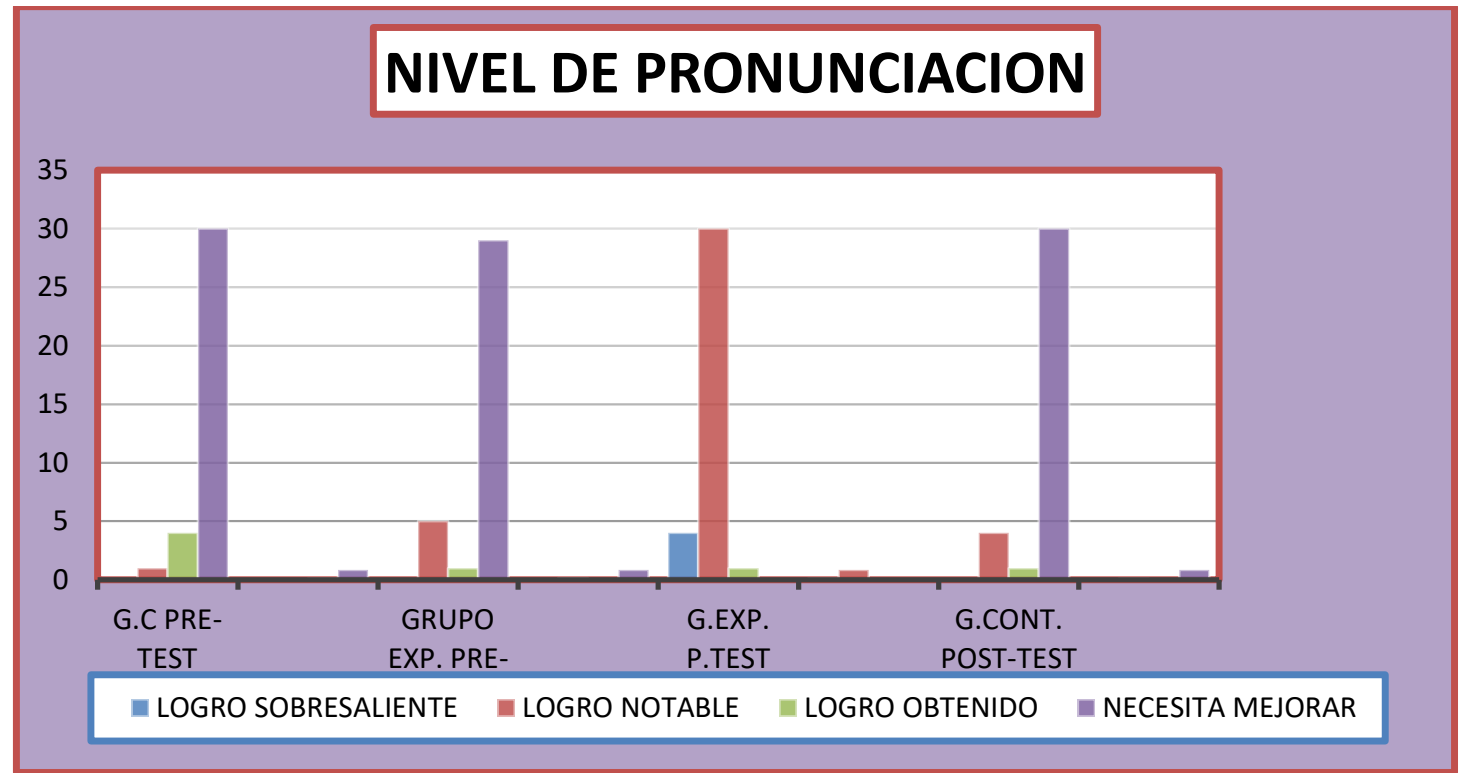

\subsection{DESCRIPCIÓN}

Los resultados de la dimensión gramática ambos grupos, compuesta por 70 se aprecia que en el grupo control antes de la aplicación de la estrategia Speaking Club on-line el 71,00\% se encuentra en nivel que necesita mejorar el $20 \%$ logro obtenido y el 2,00\% logro notable, los resultados del grupo experimental antes de la aplicación de la estrategia Speaking club on-line el 71\% está en inicio necesita mejorar, un 20\% están logro alcanzado, en logro notable un 2,90, Después de la aplicación de la estrategia Speaking Club on-line el grupo control el 60,00\% necesita mejorar, el 28,00 está en logro obtenido, el 6,00 se encuentra en logro notable y el 6,00 logro sobresaliente. En cambio, en el grupo experimental, el 3\% aun necesita mejorar y el 54\% logro obtenido un 34,00 \% logro sobresaliente. Se aprecia que el grupo experimental tiene un mejor desempeño en la prueba del post.tets. 
GRAFICO

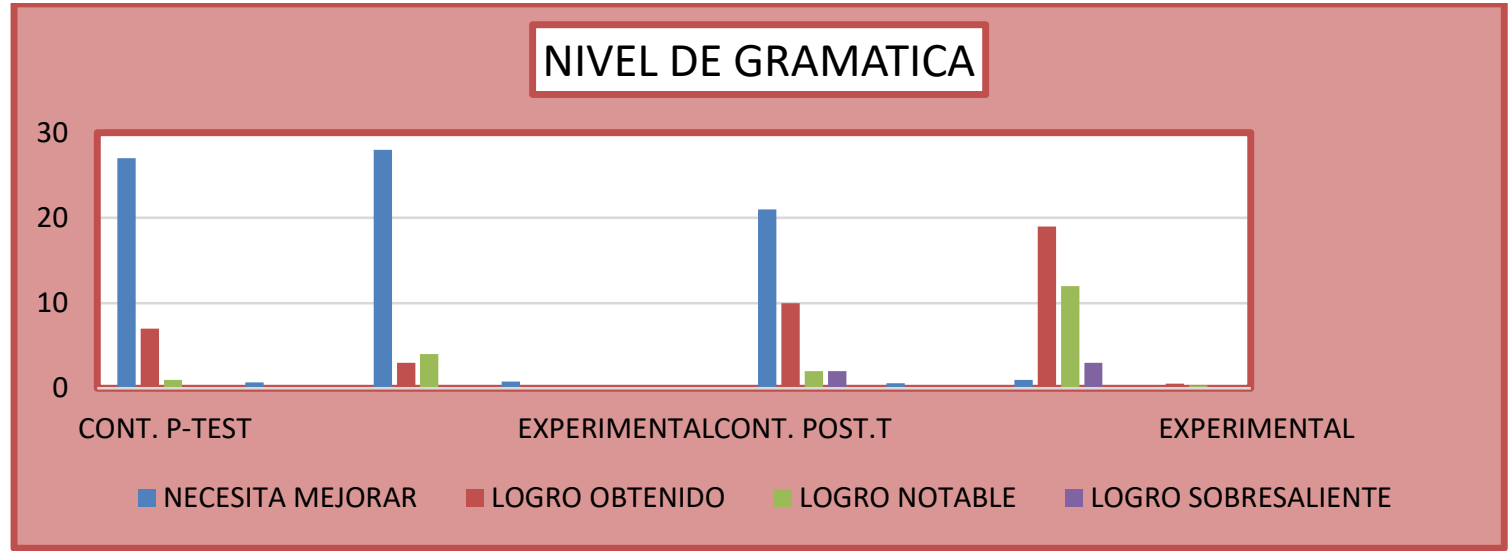

Tabla 13 Nivel de la vocabulary del idioma ingles

\begin{tabular}{|c|c|c|c|c|c|c|c|c|}
\hline \multirow[t]{2}{*}{ vocabulario } & \multicolumn{2}{|c|}{ CONT. P-TEST } & \multicolumn{2}{|c|}{ EXPERIMENTAL } & \multicolumn{2}{|c|}{ CONT. POST.T } & \multicolumn{2}{|c|}{ EXPERIMENTAL } \\
\hline & $\mathrm{n}$ & $\%$ & $\mathrm{n}$ & $\%$ & $\mathrm{n}$ & $\%$ & $n$ & $\%$ \\
\hline $\begin{array}{l}\text { NECESITA } \\
\text { MEJORAR }\end{array}$ & $85,70 \%$ & 29 & $82,90 \%$ & $80 \%$ & 18 & $51 \%$ & 1 & $3 \%$ \\
\hline $\begin{array}{l}\text { LOGRO } \\
\text { OBTENIDO }\end{array}$ & $11,40 \%$ & 1 & $2,90 \%$ & $8 \%$ & 12 & $34 \%$ & 18 & $51 \%$ \\
\hline $\begin{array}{l}\text { LOGRO } \\
\text { NOTABLE }\end{array}$ & $2,90 \%$ & 5 & $14,30 \%$ & $11,40 \%$ & 3 & $9,00 \%$ & 15 & $43,00 \%$ \\
\hline $\begin{array}{l}\text { LOGRO } \\
\text { SOBRESALIENTE }\end{array}$ & 0 & $0,00 \%$ & 0 & $0,00 \%$ & 2 & $6,00 \%$ & 1 & $3,00 \%$ \\
\hline
\end{tabular}

\subsection{DESCRIPCION}

En la Tabla 4, se aprecia que en el pretest el $85.7 \%$ de los estudiantes del grupo experimental están en el rango necesita mejorar en la Competencia Vocabulario del Idioma Inglés el 11,40\% se encuentran en promedio de logro el grupo experimental el 82,00\% está en promedio de necesita mejorar de 2,90\%; logro obtenido en ningunos de los dos grupos hay logro sobresaliente, evidenciándose que antes del desarrollo de la estrategia Speaking Club on-line, los estudiantes del grupo experimental y control presentan similares deficiencias en el nivel de vocabulario del Idioma Inglés después de aplicar la estrategia multimedia a los dos grupos el grupo control el 51,00 \% necesita mejorar, el 34,00\% logro obtenido el 9,00\% logro notable y el 6,00\% logro sobresaliente mientras que el grupo experimental el $3,00 \%$ necesita mejorar el 51,00 están en logro obtenido el 43,00 logro notable y un 3,00\% logro sobresaliente. 


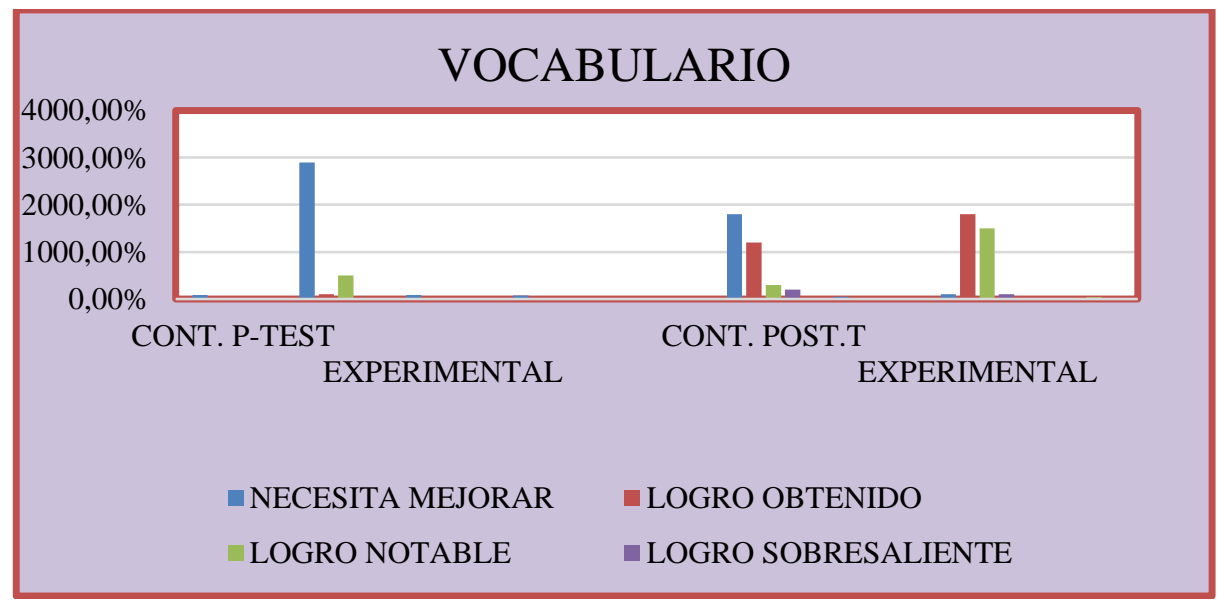

\subsection{CONTRASTACIÓN DE HIPÓTESIS}

Para probar si las variables se asemejan a una distribución normal e realizó la prueba de normalidad de hipótesis Shapiro Wilk

Tabla 14, Prueba de normalidad de Shapiro Wilk de los niveles del Idioma Inglés de los estudiantes de nivel secundaria de la Unidad Educativa Nicolás Infante Díaz

\begin{tabular}{|c|c|c|c|c|c|}
\hline \multirow[b]{2}{*}{ GRUPO } & \multirow[b]{2}{*}{ TEST } & \multirow[b]{2}{*}{ VARIABLE / DIMENSIONES } & \multicolumn{3}{|c|}{ SHAPIRO WILK } \\
\hline & & & Estadístico & gl & Sig. \\
\hline \multirow{8}{*}{$\begin{array}{l}\text { Grupo } \\
\text { Experimental }\end{array}$} & \multirow{4}{*}{$\begin{array}{l}\text { Pre } \\
\text { test }\end{array}$} & Vocabulario & 0.897 & 35 & 0.016 \\
\hline & & Pronunciación & 0.852 & 35 & 0.002 \\
\hline & & Fluidez & 0.826 & 35 & 0.001 \\
\hline & & Gramática & 0.899 & 35 & 0.017 \\
\hline & \multirow{4}{*}{$\begin{array}{c}\text { Post } \\
\text { test }\end{array}$} & Vocabulario & 0.956 & 35 & 0.343 \\
\hline & & Pronunciación & 0.915 & 35 & 0.039 \\
\hline & & Fluidez & 0.899 & 35 & 0.017 \\
\hline & & Gramática & 0.825 & 35 & 0.001 \\
\hline \multirow{8}{*}{$\begin{array}{l}\text { Grupo } \\
\text { Control }\end{array}$} & \multirow{4}{*}{$\begin{array}{l}\text { Pre } \\
\text { test }\end{array}$} & Vocabulario & 0.939 & 34 & 0.185 \\
\hline & & Pronunciación & 0.900 & 35 & 0.030 \\
\hline & & Fluidez & 0.941 & 35 & 0.205 \\
\hline & & Gramática & 0.901 & 35 & 0.031 \\
\hline & \multirow{4}{*}{$\begin{array}{l}\text { Pre } \\
\text { test }\end{array}$} & Vocabulario & 0.933 & 34 & 0.044 \\
\hline & & Pronunciación & 0.900 & 35 & 0.030 \\
\hline & & Fluidez & 0.878 & 35 & 0.011 \\
\hline & & Gramática & 0.960 & 35 & 0.481 \\
\hline
\end{tabular}

Nota: Base de datos de las dimensiones de la expresión oral Ingles Salida: SPSS Vrs. 25.0

\subsection{INTERPRETACIÓN}

Los datos se distribuyen de manera no normal; por lo cual, se decidió utilizar la prueba U de Mann Whitney, prueba no paramétrica, con el fin de contrastar las hipótesis de investigación.

\subsection{CONTRASTACIONES DE HIPÓTESIS}

H0: La implementación del Speaking-Club on-line como estrategia multimedia no mejora 
significativamente el aprendizaje de los estudiantes en la expresión oral del idioma Ingles del Distrito 12D03 Quevedo-Mocache.

H1: La implementación del Speaking-Club on-line como estrategia multimedia mejora significativamente el aprendizaje de los estudiantes en la expresión oral del idioma Ingles del Distrito 12D03 Quevedo-Mocache.

Tabla15.Contrastaciones de hipótesis

\begin{tabular}{lccccccc}
\hline \multirow{2}{*}{ Prueba } & \multicolumn{2}{c}{$\begin{array}{c}\text { Experimental } \\
(\mathbf{n}=35)\end{array}$} & \multicolumn{2}{c}{$\begin{array}{c}\text { Control } \\
(\mathbf{n}=35)\end{array}$} & \multicolumn{2}{c}{ U de Mann- Whitney } \\
\cline { 2 - 9 } & $\mathbf{N}$ & $\begin{array}{c}\text { Rango } \\
\text { promedio }\end{array}$ & $\mathbf{N}$ & $\begin{array}{c}\text { Rango } \\
\text { promedio }\end{array}$ & U & Z & Sig. \\
\hline Pre-test & 35 & 34.55 & 35 & 26.45 & 328.500 & -1.823 & 0.068 \\
Post-test & 35 & 44.68 & 35 & 16.32 & 24.500 & -6.380 & 0.000 \\
\hline
\end{tabular}

U. estadístico de Mann-Whitney

Z. valor normal estándar

p. significancia

\section{Interpretación:}

Se puede evidenciar que $\mathrm{p}<0.05$, por lo que se rechaza Ho, se asume que la aplicación de la estrategia speaking Club on-line ha tenido efectos significativos en la expresión oral del idioma ingles.

\subsection{HIPÓTESIS ESPECIFICA}

H0: La implementación del Speaking-Club on-line como estrategia multimedia no mejora significativamente en el aprendizaje de los estudiantes en el nivel de la fluidez del idioma Ingles del Distrito 12D03 Quevedo-Mocache.

H1: La implementación del Speaking-Club on-line como estrategia multimedia mejora significativamente en el aprendizaje de los estudiantes en el nivel de la fluidez del idioma Ingles del Distrito 12D03 Quevedo-Mocache.

\section{Tabla 17}

\begin{tabular}{rccccc}
\hline fluidez & Grupo & Prom & Dif & $\begin{array}{c}\text { Z } \\
\text { (U Mann } \\
\text { Whitney) }\end{array}$ & Significancia \\
\hline \multirow{2}{*}{ PreTest } & Experimental & 30.36 & & & $\mathrm{p}=0.186>0.05$ \\
\cline { 2 - 6 } & Control & 29.45 & 0.91 & 1.323 & No Significativo \\
\hline Post Test & Experimental & 49.96 & 20.19 & 5.879 & $\mathrm{p}=0.000<0.05$ \\
\cline { 2 - 6 } & Control & 29.77 & & & Significativo \\
\hline
\end{tabular}

Nota: Base de datos de las competencias en inglés (Anexo 05) Prom:

Promedio / Dif: Diferencia.

Salida: SPSS Vrs. 25.0 


\subsection{INTERPRETACIÓN}

Se puede observar que la diferencia promedio del pretest evidenciándose que después del desarrollo de la estrategia Speaking Club On-Line, los estudiantes del grupo experimental y control presentan significativas diferencias sobre la Expresión Oral del Idioma Inglés, donde el grupo experimental mejoró la Competencia por lo que se rechaza la hipótesis nula y se acepta la hipótesis alterna.

\subsection{HIPÓTESIS ESPECIFICA 1}

H0: La implementación del Speaking-Club on-line como estrategia multimedia no mejora significativamente en el aprendizaje de los estudiantes en el nivel de la fluidez del idioma Ingles del Distrito 12D03 Quevedo-Mocache.

H1: La implementación del Speaking-Club on-line como estrategia multimedia mejora significativamente en el aprendizaje de los estudiantes en el nivel de la fluidez del idioma Ingles del Distrito 12D03 Quevedo-Mocache.

Tabla. 18 Prueba de hipótesis de la estrategia multimedia Speaking Club On-line en la mejora de la Fluidez en el Idioma Inglés de los estudiantes de segundo bachillerato de la Institución Educativa Nicolás Infante Díaz, 2021

\begin{tabular}{|c|c|c|c|c|c|}
\hline fluidez & Grupo & Prom & Dif & $\begin{array}{c}Z_{c} \\
\text { (U Mann } \\
\text { Whitney) }\end{array}$ & Significancia \\
\hline \multirow{2}{*}{$\begin{array}{l}\text { Pre- } \\
\text { Test }\end{array}$} & Experimental & 9.80 & \multirow{2}{*}{0.30} & \multirow{2}{*}{1.075} & $p=0.282>0.05$ \\
\hline & Control & 9.50 & & & No Significativo \\
\hline $\begin{array}{l}\text { Post- } \\
\text { Test }\end{array}$ & Experimental & 15.08 & 5.58 & 5.823 & $p=0.000<0.05$ \\
\hline
\end{tabular}

Nota: Base de datos de las competencias en inglés (Anexo 05) Prom: Promedio /

Dif: Diferencia.

Salida: SPSS Vrs. 25.0

\subsection{INTERPRETACIÓN}

Se evidenciándose que luego del desarrollo de la estrategia Speaking Club on-line, los estudiantes del grupo experimental y control presentan mejoras destacadas sobre la fluidez en el Idioma Inglés.

\subsection{HIPÓTESIS ESPECIFICA 2}

H0: La implementación del Speaking-Club on-line como estrategia multimedia no mejora significativamente en el aprendizaje de los estudiantes en el nivel de la gramática del idioma Ingles del Distrito 12D03 Quevedo-Mocache. 
H1: La implementación del Speaking-Club on-line como estrategia multimedia mejora significativamente en el aprendizaje de los estudiantes en el nivel de la fluidez del idioma Ingles del Distrito 12D03 Quevedo-Mocache.

Tabla 19. Prueba de hipótesis de la implementación de la estrategia Speaking Club On-line en la mejora de la Gramática en el Idioma Inglés de los estudiantes de segundo bachillerato de la Institución Educativa Nicolás Infante Díaz, 2021

\begin{tabular}{cccccc} 
Gramática & Grupo & Prom & Dif & $\begin{array}{c}\text { (U Mann } \\
\text { Whitney) }\end{array}$ & Significancia \\
\hline \multirow{2}{*}{ PreTest } & Experimental & 11.16 & 0.25 & 0.781 & $\mathrm{p}=0.435>0.05$ \\
\cline { 2 - 4 } & Control & 10.91 & & No Significativo \\
\hline Post-Test & Experimental & 19.16 & 8.21 & 5.920 & $\mathrm{p}=0.000<0.05$ \\
& Control & $\mathbf{1 0 . 9 5}$ & & Significativo \\
\hline
\end{tabular}

Nota: Base de datos de las competencias en inglés (Anexo 05) Prom: Promedio /

Dif: Diferencia.

Salida: SPSS Vrs. 25.0

\subsection{INTERPRETACIÓN}

Se visualiza que la diferencia promedio del post-test con valor de la prueba estadística es $=$ 5.920 y nivel de significancia menor al 5 evidenciándose que luego de la aplicación de la estrategia los estudiantes del grupo experimental y control mejoraron en la gramática en el Idioma Inglés.

\subsection{HIPÓTESIS ESPECÍFICA 3}

H0: La implementación del Speaking-Club on-line como estrategia multimedia no mejora significativamente en el aprendizaje de los estudiantes en el nivel de la gramática del idioma Ingles del Distrito 12D03 Quevedo-Mocache.

H1: La implementación del Speaking-Club on-line como estrategia multimedia mejora significativamente en el aprendizaje de los estudiantes en el nivel de la fluidez del idioma Ingles del Distrito 12D03 Quevedo-Mocache.

Tabla 21. Prueba de hipótesis del Programa Speaking Club On-line en la mejora de Vocabulario en el Idioma Inglés de los estudiantes de segundo de bachillerato de la Institución Educativa Nicolás Infante Díaz

\begin{tabular}{cccccc} 
Gramática & Grupo & Prom & Dif & $\begin{array}{c}\text { (U Mann } \\
\text { Whitney) }\end{array}$ & Significancia \\
\hline \multirow{2}{*}{ PreTest } & Experimental & 9.40 & 0.35 & 1.122 & $\mathrm{p}=0.435>0.05$ \\
& Control & 9.05 & & & No Significativo \\
\hline \multirow{2}{*}{ Post-Test } & Experimental & 15.72 & 9.32 & 5.860 & $\mathrm{p}=0.000<0.05$ \\
& Control & 16.40 & & & Significativo \\
\hline
\end{tabular}




\subsection{INTERPRETACIÓN}

Se puede apreciar que la diferencia promedio del post-test es con valor de la prueba estadística $=5.80$ y nivel de significancia menor al $5 \%(\mathrm{p}<0.05)$, evidenciándose que luego del desarrollo de la aplicación de la estrategia Speaking Club On-line, los estudiantes del grupo experimental y control presentan marcadas mejoras sobre el Vocabulario en el Idioma Inglés.

\section{DISCUSION}

Actualmente, la implementación de la estrategia multimedia Speaking Club on-line ha adquirido gran relevancia en las instituciones educativas, esto constituye una herramienta de trabajo interactiva que permite que los estudiantes interactúen mediante los recursos tecnológicos, en la presente investigación tuvo como objetivo general determinar si la implementación de la estrategia Speaking club on-line mejora significativamente la expresión oral en los estudiantes del nivel secundaria de la IE Nicolás Infante Díaz. Para dar inicio a la discusión de los resultados, se parte de los hallazgo encontrados en el proceso investigativo.

Ahora bien es necesario identificar cual es el nivel de los estudiantes en la expresión oral del idioma inglés, de los resultados obtenidos en el Pre-test el 85,7\% de los estudiantes de grupo control se encuentran en niveles que necesitan mejorar, el 11,4\% en nivel logro obtenido y un $2 \%$ en nivel de logro notable y el grupo experimental el $82,9 \%$ en similares condiciones en nivel de necesita mejorar, el 2,9 en nivel de logro obtenido y un $14 \%$ en logro notable de los resultados obtenido que los podemos ver en la tabla 6 y en el grafico 1, ambos grupos se encuentran en condiciones similares en los niveles de necesita mejorar un alto porcentaje y un porcentaje menor alcanzan niveles de logro obtenido, lo que demuestra que ambos grupos de estudiantes de la I.E tienen deficiencia en el idioma Ingles.

Los resultados obtenidos en los niveles de la expresión oral relacionan con los resultados de resultados del Muñoz, 2019, Programa TPACK para mejorar competencias lingüísticas del idioma Inglés, en esta investigación, los estudiantes del grupo experimental obtuvieron $96 \%$ nivel en el logro de las competencias de Ingles que reflejaron nivel C, lo que demuestra que la implementación de speaking club como estrategia influye significativamente en estudiantes con deficiencias en el idioma ingles

En otro orden de ideas, en el segundo objetivo específico. Evaluar los niveles de aprendizaje de los estudiantes, en la expresión oral del idioma Ingles, después de la implementación del Speaking-Club on-line como estrategia multimedia., observándose que 
luego de la implementación de la estrategia multimedia los estudiantes en el grupo experimental el 9,00\% está en nivel de logro sobresaliente, un 37,00\% logro notable, un 51\% alcanzo nivel de logro obtenido y los resultados evidencia que un 3,00\% necesita mejorar, como se puede evidenciar se presenta cambios positivos y un incremento en el nivel del idioma inglés antes un alto porcentaje se encontraba en necesita mejorar y mejoraron en nivel ubicándose en nivel logro notable y sobresaliente ( Tabla 7 ).Los resultados se pueden contrastar con los presentados por Torres, (2016) quien en su investigación determino la influencia de la tecnología en el desarrollo de las competencias comunicativas (expresión oral) en 130 estudiantes logro evidenciar que el uso de la tecnología si influye en las competencias comunicativas en los estudiantes resultado 11,85 a diferencia del otro grupo 16,95. Esto quiere decir que se puede emplear estrategias interactivas como herramienta Tics para el aprendizaje de un segundo idioma.

\section{CONCLUSIONES}

Speaking Club on-line como estrategia multimedia mejora significativamente la expresión oral del Idioma Inglés en estudiantes de la I.E Nicolás Infante Díaz, Quevedo 2020. Teniendo en cuenta que el rango promedio Pre-Test es de 34.55 y después de la implementación de la estrategia multimedia en el Post-Test 44.68 con un valor p menor al nivel de significancia $=0.05$, se puede evidenciar que existe una influencia significativa para la aceptación de la hipótesis de la investigación.

Speaking Club On-line como estrategia multimedia mejorar significativamente la fluidez en el idioma inglés, los niveles antes de la implementación de la estrategia se encontraban en $82,00 \%$ en inicio nivel de necesita mejorar y un $14 \%$ logro obtenido después de la aplicación los niveles presentan incremento el 3\% necesita mejorar, el $51 \%$ logro obtenido. Los estudiantes mejoraron significativamente la fluidez del idioma inglés.

Speaking Club On-line como estrategia multimedia mejorar significativamente la Gramática en el idioma inglés, los niveles antes de la implementación de la estrategia se encontraban en $82,00 \%$ en inicio nivel de necesita mejorar y un 14\% logro obtenido después de la aplicación los niveles presentan incremento el 3\% necesita mejorar, el $51 \%$ logro obtenido. Los estudiantes mejoraron significativamente la fluidez del idioma inglés.

Speaking Club On-line como estrategia multimedia mejorar significativamente el Vocabulario en el idioma inglés, los niveles antes de la implementación de la estrategia se encontraban el $71 \%$ está en inicio necesita mejorar, un 20\% están logro alcanzado, en logro 
notable un 2,90. Después de la aplicación de la estrategia Speaking Club on-line 3\% aún necesita mejorar y el 54\% logro obtenido un 34,00 \% logro sobresaliente.

Los estudiantes, en su mayoría, manifiestan que se encuentran satisfechos respecto a la implementación de Speaking Club on-line como estrategia multimedia para mejorar la expresión oral y también niveles del idioma : fluidez, pronunciación, gramática y vocabulario porque consideran que les ha fortalecido el speaking, ya que los temas desarrollados en las sesiones se pudo alcanzar el nivel sobresaliente, en el aspecto personal, también contribuyo porque incrementaron en la habilidad comunicativa con personas de habla inglés, de esta manera se evidenció un avance significativo en el idioma inglés en estudiantes de secundaria. 


\section{REFERENCIAS}

Anampa, F., Bolaños, R., \& Villamar, J. (2017). Fundamentos epistemológicos para orientar el desarrollo del conocimiento. Universidad Politecnica Salesiana de Ecuador.

Barturén, N. (2019). El aprendizaje basado en tareas como método para desarrollar la expresión oral en estudiantes de inglés de un Instituto Superior Tecnológico de Lima. Universidad Peruana Cayetano

Heredia. http://repositorio.upch.edu.pe/handle/upch/5958

Beltrán, M. (2017). La producción oral y comprensión del inglés como segunda lengua dentro de un sistema de inmersión en estudiantes de Educación Básica Media. Universidad de San Francisco de Quito.

British Council. (2015). Inglés en el Perú. Un análisis de la política, las percepciones y los factores de influencia. (Educationa).

https://www.britishcouncil.pe/sites/default/files/english_in_peru_may_2015.pdf

Byrne, D. (1989). Teaching oral English (Revolucion).

Carbajal, Z. (2013). Enseñanza del inglés en secundaria: una propuesta innovadora. Revista Eduación.

Chérrez, A. (2017). The Use of Drama-Oriented Activities to Improve Oral Fluency in an EFL Classroom at Politécnica Salesiana University. (Vol. 4). http://dspace.ucuenca.edu.ec/handle/123456789/26603

Chomsky, N. (1965). Aspects of the Theory of Syntax. Massachusetts Institute of Technology. https://doi.org/10.1017/S0022226700012998

Domínguez, J. (2016). Manual de metodología de la investigación científica.

ULADECH Católica.

Fink, A. (1995). Evaluation for education and psychology. (Sage Publi). https://psycnet.apa.org/record/1995-97751-000

Flores, M. (2017). Estrategias didácticas que inciden en el desarrollo de la expresión oral para el aprendizaje significativo del idioma inglés a nivel intermedio en egresados gestiones 2014 y 2015 de la Escuela Superior de Formación de maestros "Simón Bolívar". Universidad Mayor de San Andrés. http://repositorio.umsa.bo/xmlui/handle/123456789/14202

Harmer, J. (2000). The practice of English Language Teaching. (Fourth edi). LongmanPerson.https://www.academia.edu/25472823/The_Practice_of_English_Language_Te aching_4th_Edition_Jeremy_Harmer

Luchini, P., \& García, M. (2015). Sobre el grado de acento extranjero y fluidez en la clase de pronunciación inglesa: un estudio evaluativo. 193-213. https://doi.org/10.22456/2238-8915.51722 
Manrique, S. (2018). Estrategias de aprendizaje cooperativo y sus efectos en la expresión oral del idioma inglés de los estudiantes de la Universidad Católica

Olivares, G. (2017). El uso de estrategias para desarrollar la expresión oral en inglés en estudiantes de segundo semestre de bachillerato del CECyTE Tecamachalco.Universidad Iberoamericana Puebla. http://hdl.handle.net/20.500.11777/3343

Oxford, R. (1990). Language Learning Strategies: What Every Teacher Should Know (pp. 140143). https://core.ac.uk/download/pdf/29197062.pdf

Pérez, R. (2000). LA EVALUACIÓN DE PROGRAMAS EDUCATIVOS:CONCEPTOS BÁSICOS, PLANTEAMIENTOSGENERALES Y PROBLEMÁTICA. In Revista De Investigación Educativa (Vol. 18, pp. 261-287). Universidad de Murcia. https://revistas.um.es/rie/article/view/121001

Ramírez, S., \& Artunduaga, M. (2017). Authentic Tasks to Foster Oral Production Among English as a Foreign Language Learners. Reportes de Ivestigación.

Rivera, G. (2016). Desarrollo del enfoque comunicativo y el aprendizaje del idioma inglés en los estudiantes de la Universidad Jaime Bausate y Mesa.http://hdl.handle.net/20.500.11818/1105

Ruiz, A., García, L., \& Díaz, A. (2007). Alternativa curricular para favorecer el desarrollo de la expresión oral en los alumnos de la escuela primaria. (Universita). Instituto Central de Ciencias Pedagógicas. https://www.academia.edu/8351633/Alternativa_curricular_para_favorecer_el_ desarrollo_de_la_expresión_oral 